\title{
Lattice disorder and magnetism in $f$-electron intermetallics
}

\author{
C. H. Booth ${ }^{\text {a,* }}$, S.-W. Han ${ }^{\text {a,b }}$, S. Skanthakumar ${ }^{\text {, }}$,J. L. Sarrao ${ }^{\text {d }}$ \\ ${ }^{a}$ Chemical Sciences Division, MS 70A-1150, Lawrence Berkeley National Laboratory, Berkeley, CA 94720, USA \\ b Chonbuk National University, Jeonju, 561-756, Korea \\ ${ }^{\mathrm{c}}$ Chemistry Division, Argonne National Laboratory, Argonne, IL 60439, USA \\ d Materials Sciences Division, Los Alamos National Laboratory, Los Alamos, NM 87501, USA
}

\begin{abstract}
Real materials can have real differences compared to ideal systems. For instance, non-Fermi liquid (NFL) behavior was initially thought to be due to chemical disorder, since the first such materials were all substituted. Although several nominally well-ordered NFL's have been discovered and extensively studied, the effect of disorder on the magnetic properties of $f$-electron intermetallic systems remains poorly understood. Disorder in NFL systems is reviewed from an experimental, local structure point of view, including a discussion of results on the nominally ordered $\mathrm{U}_{3} \mathrm{Ni}_{3} \mathrm{Sn}_{4}$ and $\mathrm{CeCoIn}$ systems, and the chemically disordered $\mathrm{UCu}_{4} \mathrm{Pd}$ and $\mathrm{CeRhRuSi}_{2}$ systems.
\end{abstract}

Key words: non-Fermi liquids, lattice disorder, pair-distribution functions, extended x-ray absorption fine structure PACS: 72.15.Qm, 61.10.Ht, 71.23.-k, 71.27.+a

\section{Introduction}

Recent interest in non-Fermi liquid (NFL) properties of $f$-electron intermetallics has been overwhelming due to both the possibility of studying a new state of matter and because of the potential applications to similar problems in the hightemperature superconductors [1]. Although several ambient-pressure NFL materials have been discovered recently that appear to be structurally very well ordered (eg. $\mathrm{YbRh}_{2} \mathrm{Si}_{2}[2], \mathrm{U}_{3} \mathrm{Ni}_{3} \mathrm{Sn}_{4}[3,4]$, and $\mathrm{CeCoIn}_{5}[5]$ ), the originally discovered NFL materials are all disordered, typically by chemical substitution [1]. This observation germinated several

\footnotetext{
* Corresponding author. Tel.: +1-510-486-6079; fax: +1510-486-5596; email: chbooth@lbl.gov
}

theoretical and experimental studies on the effects of disorder on $f$-electron interactions. For instance, Bernal et al. [6] found that, despite the apparent order in $\mathrm{UCu}_{4} \mathrm{Pd}$, the Knight shift, magnetic susceptibility $\chi(T)$ and heat capacity could all be described by assuming a so-called Kondo disorder model (KDM) where only Kondo interactions are considered but a distribution $P\left(T_{\mathrm{K}}\right)$ of Kondo temperatures $T_{\mathrm{K}}$ exists that extends down to very low temperatures. These concepts were given a more rigorous foundation by Miranda et al. [7] and extended in various studies [8].

Despite the theoretical work on disorder models of NFL behavior, few systems have behavior that can be well described within such a framework. In this paper, we describe our work attempting to better characterize local lattice disorder and relate the 
results to the KDM. To date, we have focused on several NFL's: the nominally ordered $\mathrm{CeCoIn}_{5}$ and $\mathrm{U}_{3} \mathrm{Ni}_{3} \mathrm{Sn}_{4}$ systems, and the nominally disordered $\mathrm{CeRhRuSi}_{2}$ and $\mathrm{UCu}_{4} \mathrm{Pd}$ systems. Taken together, these results do not favor disorder-based models of NFL behavior as a universal mechanism, although disorder can be seen to play a role, particularly in $\mathrm{UCu}_{4} \mathrm{Pd}$.

\section{The KDM and local structural disorder}

Although we are primarily concerned with whether real NFL materials exhibit local structure disorder, we must ask ourselves whether any measured disorder (or upper limits of disorder) can explain NFL behavior without resorting to a breakdown of the quasiparticle picture of Fermi liquid theory. As a simplifying assumption, we focus only on Kondo interactions. In a KDM, once one has $P\left(T_{\mathrm{K}}\right)$ one can calculate the expected $\chi(T)$ and heat capacity, assuming a particular crystal field scheme, within the Bethe ansatz [9]. One calculates $P\left(T_{\mathrm{K}}\right)$ by starting with a typical form for the Kondo temperature:

$T_{\mathrm{K}}=E_{\mathrm{F}} e^{-\frac{\epsilon_{f}}{\rho(0) V_{f c}^{2}}}$,

where $E_{\mathrm{F}}$ is the Fermi energy, $\epsilon_{f}$ is the bare $f$-level energy, $\rho(0)$ is the density of states at the Fermi level, and $V_{f c}$ is the $f$-electron/conduction electron hybridization matrix element. Disorder can be applied to this model in several ways. First, although $E_{\mathrm{F}}$ is calculable assuming an infinitely periodic lattice, it is actually a local quantity and can vary widely across a crystal, for instance, in the vicinity of a vacancy or near the surface. Similarly, $\rho(0)$ and $\epsilon_{f}$ can also vary widely for similar reasons. However, $V_{f c}$ is a truly local quantity that only depends on the degree of overlap between the $f$-shell and the conduction electrons and their orbital symmetries.

The variant of the KDM considered here (referred to as the Kondo lattice disorder model, or KLDM) utilizes a tight-binding approach to compute $V_{f c}$. Since the $d$-electrons represent the main contribution to the conduction band in the materials considered here, we only consider the $V_{f d}$ ma- trix element. The tight-binding formalism for each pair of atoms involving the $f$-ions and the neighboring atoms contributing $d$ electrons to the conduction band gives [10]:

$V_{f d}=\sum_{\text {pairs }} \frac{\eta_{f d} \hbar^{2}}{m_{e}} \frac{\sqrt{\left(r_{f}^{5} r_{d}^{3}\right)}}{R_{f-d}^{6}}$,

where $r_{l}$ is the radius of the electronic shell of the atom contributing the states with angular momentum $l$ (=d or $f$ ), $R_{f-d}$ is the bond length between the $f$-ion and $d$-contributing atoms, and $\eta_{f d}$ is a factor which only depends on the $l$ 's and bond symmetry. Within this approach, $V_{f c}$ is often larger than experimentally observed by a factor of two. Other short comings of this approach include using a constant for $E_{\mathrm{F}}, \rho(0)$, and $\epsilon_{f}$, whereas these quantities depend on the local disorder, and the sensitivity of $V_{f c}$ to phasing of the overlapping shells. In any case, the model can absorb some systematic differences in the calculated $V_{f c}$ by allowing $r_{K}=\rho(0) / \epsilon_{f}$ to be a fitting parameter.

Lattice disorder is included in Eq. 2 with a static bond length distribution $P\left(R_{f-d}\right)$, and by varying the neighboring species via the $r_{l}$ 's. The latter produces a discrete $P\left(V_{f c}\right)$ distribution, whereas the former creates a continuous distribution. Although discrete disorder can produce a fairly wide $P\left(V_{f c}\right)$, only continuous disorder can generate enough low$T_{\mathrm{K}}$ moments to obtain NFL behavior.

This model is demonstrated in Figs. 1 and 2. Figure 1 shows $\chi(T)$ data for the CeRhRuSi ${ }_{2}$ sample discussed in Sec. 4, plotted as a function of $\log (T)$ to demonstrate the NFL low temperature divergence. The fit uses the KLDM with angular momentum $J=3 / 2, E_{F}=7720 \mathrm{~K}, r_{K}=0.055 \mathrm{eV}^{-2}$ and $\sigma_{\text {static }}^{2}=0.0064 \AA^{2}$. The corresponding distribution of $V_{f c}$ 's and $T_{K}$ 's are shown in Fig. 2. Note that $P\left(T_{K}\right)$ continues to grow at low temperatures, but necessarily goes to zero at $T_{K}=0 \mathrm{~K}$. The value of $\sigma_{\text {static }}^{2}$ is in fact a lower limit since the Si $p$ electrons also contribute to the conduction band in this system, and will therefore increase $V_{f c}$, which will in turn force even more severe broadening to generate the NFL behavior.

The methods employed here for determining lattice disorder are intentionally not the usual methods of single crystal and powder diffraction 


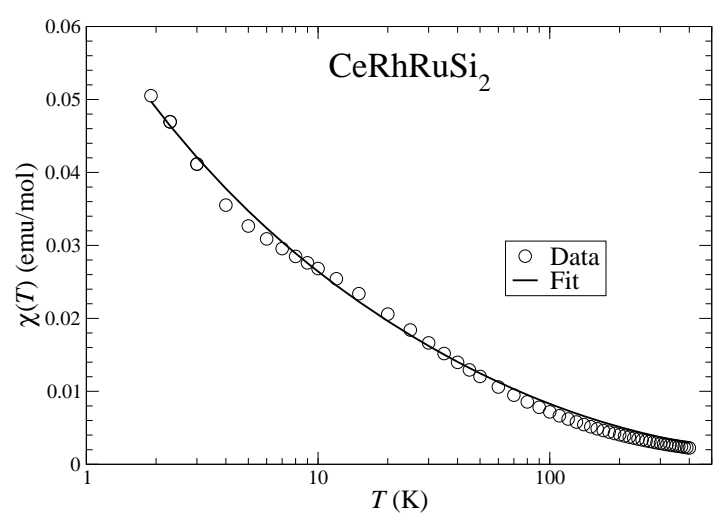

Fig. 1. Magnetic susceptibility $\chi(T)$ data for $\mathrm{CeRhRuSi}_{2}$. The solid line is a fit to the KLDM (see text).

measurements coupled with Rietveld analysis. Rather, we employ the local structure techniques of extended x-ray absorption fine structure (EXAFS) analysis and pair-distribution function (PDF) analysis of powder diffraction data to look for systematic differences between the long-range, average structure given by Rietveld analysis and the short-range, local structure given by EXAFS and PDF.

In order to directly compare to the KLDM, however, we need a more quantitative approach. For this purpose, we perform temperature-dependent EXAFS measurements of the pair-distance distribution variances, $\sigma^{2}$ 's. With a correlated-Debye model [11], we estimate the static (i.e. nonthermal) contribution $\left(\sigma_{\text {static }}^{2}\right)$ from the $f$-ion's

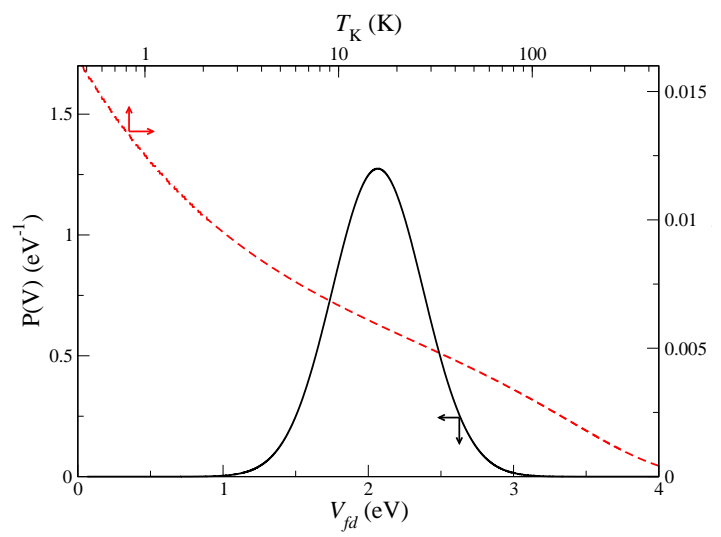

Fig. 2. The distribution of hybridization strengths $P\left(V_{f d}\right)$ (solid) and the distribution of Kondo temperatures $P\left(T_{\mathrm{K}}\right)$ (dashed) used for the fit in Fig. 1. nearest neighbor $\sigma^{2}$ 's. For instance, in the case of $\mathrm{CeRhRuSi}_{2}$, the main contribution to the conduction band is from the $\mathrm{Rh} / \mathrm{Ru}$ sites, and so we concentrate on the eight nearest $\mathrm{Ce}-\mathrm{Rh} / \mathrm{Ru}$ pairs at $\sim 2.9 \AA$. These pairs are also the most likely to be disordered, since each $\mathrm{Rh} / \mathrm{Ru}$ site is randomly occupied by either Rh or Ru. These $\sigma^{2}$ 's are then fit to:

$\sigma_{\text {meas }}^{2}\left(T, \theta_{\mathrm{cD}}\right)=\sigma_{\text {static }}^{2}+F\left(T, \theta_{\mathrm{cD}}\right)$,

where the $T$-dependent part $F\left(T, \theta_{\mathrm{cD}}\right)$ is given by the correlated-Debye model, which utilizes a quadratic density of states similarly to the (single site) Debye model, but substitutes the reduced mass $\mu$ of the atom pair rather than the individual atom masses and uses an extra term to account for the correlated motions of the atom pairs [11]. The model has been shown to work reasonably well for a variety of systems, but especially for simpler crystal structures [12]. The obtained values of $\sigma_{\text {static }}^{2}$ are taken as a generic measure of lattice disorder that is not fit by the structural model, and can be compared to those $\left(\sigma_{\mathrm{KDM}}\right)$ necessary for the KLDM to describe the $\chi(T)$ data.

\section{Well-ordered non-Fermi liquids: $\mathrm{U}_{3} \mathrm{Ni}_{3} \mathrm{Sn}_{4}$ and $\mathrm{CeCoIn} 5$}

Although $\mathrm{U}_{3} \mathrm{Ni}_{3} \mathrm{Sn}_{4}$ may have a low-lying Fermi liquid ground state [13], both $\mathrm{U}_{3} \mathrm{Ni}_{3} \mathrm{Sn}_{4}$ and $\mathrm{CeCoIn}_{5}$ belong to the small class of structurally well-ordered, stoichiometric materials that exhibit NFL behavior to very low temperatures. However, it is still possible that some pathological disorder that wasn't detectable using standard diffraction techniques exists. Therefore, partially as a way of verifying our techniques on well-ordered materials and to check for local structure disorder, we have measured the EXAFS as a function of temperature from both materials, and measured the x-ray $\mathrm{PDF}$ at $20 \mathrm{~K}$ for $\mathrm{CeCoIn}_{5}$. The EXAFS results on $\mathrm{U}_{3} \mathrm{Ni}_{3} \mathrm{Sn}_{4}$ have been published previously [4].

$\mathrm{X}$-ray diffraction data on $\mathrm{CeCoIn}_{5}$ were collected and the structure factor $S(q)$ was determined. The Fourier transform of $q(S(q)-1)$ between 1.0-32 $\AA^{-1}$ gives the PDF function $G(r)$ 


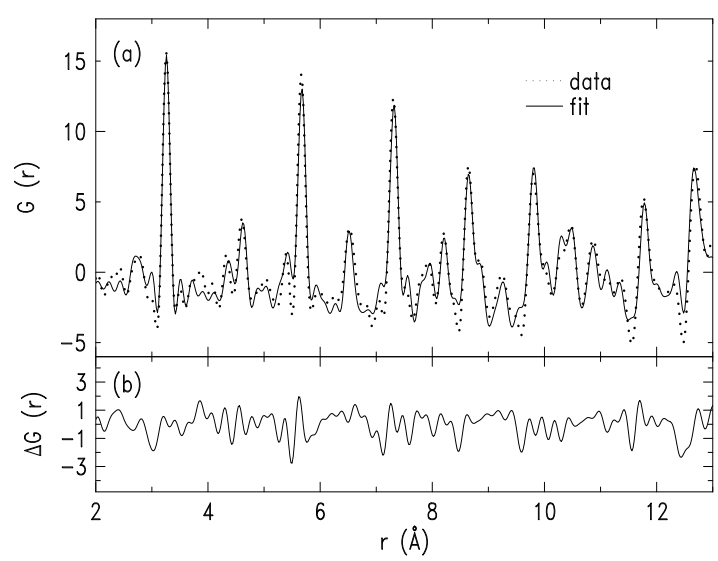

Fig. 3. (a) Pair-distribution function (PDF) $G(r)$ and fit for $\mathrm{CeCoIn}_{5}$. Panel (b) shows the residual from the fit.

as displayed in Fig. 3. The data reduction and fit were performed between 2-13 $\AA$ using the PDFFIT program [14]. This treatment is similar to conventional Rietveld refinement in that the usual parameters (lattice and positional parameters, the mean-squared displacement $u^{2}$ 's, etc.) are varied assuming a particular lattice symmetry, in this case $P 4 / m m m$ [15].

Here we look for only a few signs of disorder. The first is whether parameters that give high quality fits to the data at large $r$ also produce good fits to the near-neighbor scattering shells. This situation is clearly evident from the fit in Fig. 3, as the residual does not show any obvious $r$-dependence. Note that the first major peak at $\sim 3.26 \AA$ (due to many overlapping In-In and Ce-In paths) is not the nearest-neighbor shell in this system. There are actually two comparatively rare scattering paths at $2.73 \AA[\operatorname{In}(2)-\mathrm{Co}]$ and $\sim 2.91 \AA[\operatorname{In}(2)$ $\operatorname{In}(2)]$. Another important characteristic of the data from a well-ordered material is small $u^{2}$ 's. We obtain 0.0014(3) $\AA^{2}$ for the Ce site, 0.0028(3) $\AA^{2}$ for the Co site and 0.00269(5) $\AA^{2}$ for both In sites (constrained together). All other fit parameters are consistent with previous Rietveld results [15]. Finally, the temperature dependence of the $\sigma^{2}$ 's from EXAFS data on the related $\mathrm{CeIr}_{1-x} \mathrm{Rh}_{x} \mathrm{In}_{5}$ series shows no measurable static disorder [16]. Taking all these results together, we conclude that $\mathrm{CeCoIn}_{5}$ is, indeed, structurally well ordered.

A similar case can be made for the $\mathrm{U}_{3} \mathrm{Ni}_{3} \mathrm{Sn}_{4}$

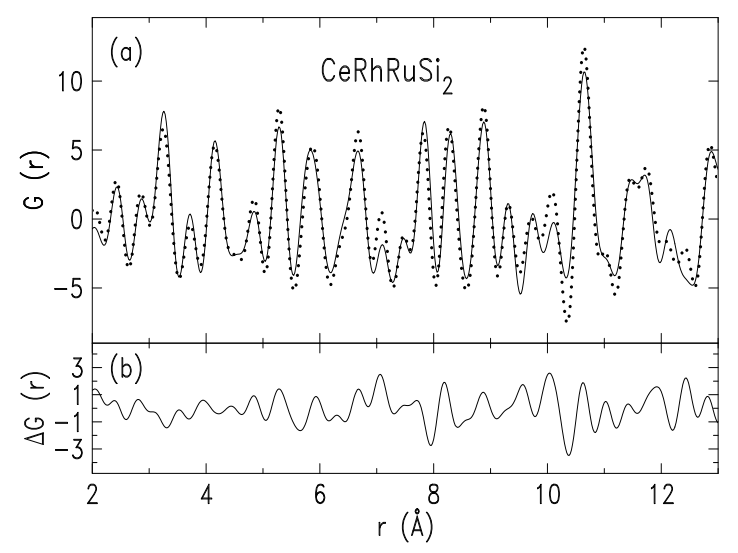

Fig. 4. (a) Pair-distribution function (PDF) $G(r)$ and fit for $\mathrm{CeRhRuSi}_{2}$. Panel (b) shows the residual from the fit.

system. Single crystals of this material form, and diffraction results on these crystals indicate a wellordered system [3]. In a recent paper [4], we reported EXAFS measurements on both single crystal and polycrystal samples, at various temperatures and stoichiometries. Similarly to the $\mathrm{CeCoIn}_{5}$ case presented above, we found no indication of lattice disorder. This conclusion was based on three factors. First, all fits were of high quality. Second, single crystal and polycrystal data and fit results were very similar to each other. Lastly, and most importantly, the correlated-Debye model fits indicated no static lattice disorder (eg. average of U-Ni and $\mathrm{Ni}-\mathrm{U}$ results give $\left.\sigma_{\text {static }}^{2}=-0.0003(6) \AA^{2}\right)$.

\section{Disordered non-Fermi liquids: $\mathrm{CeRhRuSi}_{2}$ and $\mathrm{UCu}_{4} \mathrm{Pd}$}

$\mathrm{CeRhRuSi}_{2}$ belongs to the family of cerium intermetallic compounds that have the $\mathrm{ThCr}_{2} \mathrm{Si}_{2}$ crystal structure. This material has always been placed in the chemically-disordered class of NFL materials because of the presumed random occupation of $\mathrm{Rh}$ and $\mathrm{Ru}$ on the $4 d$ site in its crystal structure's $I 4 / \mathrm{mmm}$ space group. Moreover, this system has several properties that can be modeled within the KDM [17]. However, recent spin resonance studies indicate that the low-frequency spectral weigh in the spin fluctuation spectrum is insufficient to allow the KDM to work [18]. 
We have performed both PDF and temperaturedependent EXAFS measurements of CeRhRuSi ${ }_{2}$. PDF data and fit results are displayed in Fig. 4. The data are transformed and fit over the range $0.5-18.0 \AA^{-1}$ in $q$ and $2-13 \AA$ in $r$. Fit quality is high and not a strong function of $r$ up to the maximum fitted $r$. However, the fit quality is not as high as, say, the CeCoIn 5 case presented above, and worsens somewhat at higher $r$. This situation may be due to some distortion relative to the assumed $I 4 / \mathrm{mmm}$ symmetry. In any case, the measured single-site $u^{2}$ 's at $20 \mathrm{~K}$ remain low: 0.00036(1) $\AA^{2}$, 0.003(1) $\AA^{2}$, and $0.0029(3) \AA^{2}$ for the Ce, Rh/Ru and Si sites, respectively. All other fit parameters are consistent with previous Rietveld results on the end members of the $\mathrm{Ce}\left(\mathrm{Rh}_{1-x} \mathrm{Ru}_{x}\right)_{2} \mathrm{Si}_{2}$ series [19].

Fit quality to the EXAFS data is generally very high, and the data fit the correlated-Debye model well. The Ce- Rh/Ru fits indicate $\Theta_{\mathrm{cD}}=250(20) \mathrm{K}$ and $\sigma_{\text {static }}^{2}=-0.0003(2) \AA^{2}$. Therefore both the PDF and the EXAFS results indicate a wellordered local structure, apart from the chemical disorder on the $\mathrm{Rh} / \mathrm{Ru}$ site. Moreover, these measurements preclude the KLDM shown in Figs. 1 and 2 , since that model required at least $0.0064 \AA^{2}$ of static pair-distance disorder.

The last example we present here is that of $\mathrm{UCu}_{4} \mathrm{Pd}$. These results have been published previously [20-22]. To summarize, although the system was originally thought to be well ordered, a significant fraction (order of $25 \%$ ) of the Pd atoms were found to reside on the nominally $\mathrm{Cu}$ sites. Our initial interpretation was that the measured chemical disorder would precipitate the pair-distance disorder required by the KLDM (on the order of $0.0025 \AA^{2}$ in this case), but subsequent measurements indicated such disorder was not present.

Although the initial measurements were consistent with no local disorder apart from the $\mathrm{Pd} / \mathrm{Cu}$ site interchange, the heat capacity was observed to change as a function of sample annealing time [23]. EXAFS measurements on these annealed samples indicated that the amount of site interchange was reduced after only one day of annealing, but not with more annealing. However, changes were observed in the EXAFS that were only consistent with a very small amount of $\mathrm{U}-\mathrm{Cu}$ static pairdistance disorder being slowly removed with fur-

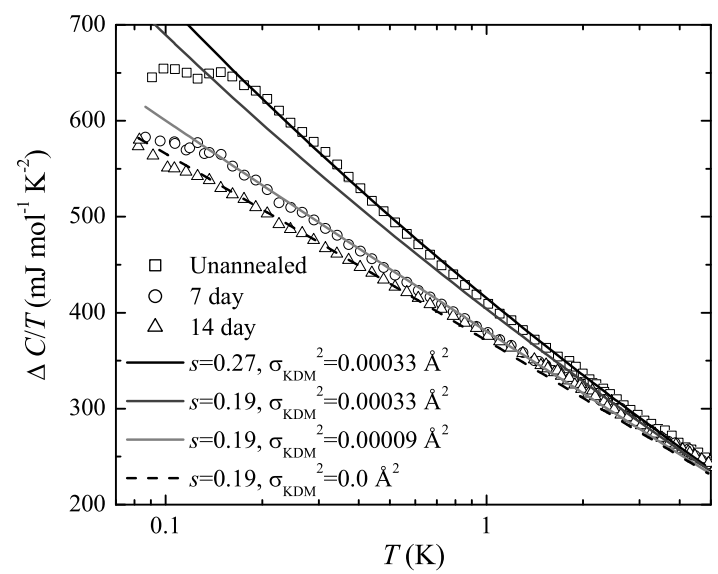

Fig. 5. Data from Ref. [22] showing both how the heat capacity of $\mathrm{UCu}_{4} \mathrm{Pd}$ changes with annealing time, and parameters in a KLDM model used to fit the data. All fits use an additional, fixed width to $P\left(T_{\mathrm{K}}\right)$ set by the 14-day anneal data. Additional width from $\sigma_{\mathrm{KDM}}^{2}$ and changes to $s$ (the fraction of $\mathrm{Pd}$ on $\mathrm{Cu}$ sites) are consistent with changes to $\sigma^{2}$ and $s$ as measurement by the EXAFS data.

ther annealing. Interestingly, the changes in the heat capacity data could be explained as due to this gradual ordering by assuming a KLDM where most of the width in $P\left(T_{K}\right)$ is due to some unexplained source (Fig. 5). This result is important because it shows that in order to understand the actual degree of divergence, the lattice disorder must be taken into account. Unfortunately, it is rarely possible to measure lattice disorder to such high precision. The $\mathrm{UCu}_{4} \mathrm{Pd}$ system is fortuitous in that the annealing dependence of the disorder was so strong. Our attempts to measure such an annealing dependence in $\mathrm{CeRhRuSi}_{2}$, for instance, have shown no change in the divergence of the heat capacity or in the local lattice order.

\section{Discussion and Conclusion}

As expected, the so-called well-ordered NFL's CeCoIn 5 and $\mathrm{U}_{3} \mathrm{Ni}_{3} \mathrm{Sn}_{4}$ show no abnormal local structure behavior, either from their temperature dependence or by comparison with the long-range structures obtained from previous Rietveld analyses. In the absence of more precise measurements, we conclude that these systems are, indeed, struc- 
turally well ordered. It is tempting to infer the same conclusion for other systems, such as $\mathrm{YbRh}_{2} \mathrm{Si}_{2}$.

Somewhat surprisingly, the chemically disordered systems $\mathrm{CeRh}_{2} \mathrm{Si}_{2}$ and $\mathrm{UCu}_{4} \mathrm{Pd}$ display very little, if any, bond length disorder. A clear case for any bond length disorder has only been made for $\mathrm{UCu}_{4} \mathrm{Pd}$ samples that have not been completely annealed. In both systems, any disorder is far below the amount required for a KLDM to describe the magnetic data. However, it is clear in the $\mathrm{UCu}_{4} \mathrm{Pd}$ system that bond-length disorder affects the divergences in $\chi(T)$ and $C / T$ : more lattice disorder creates a faster low-temperature divergence. Although a similar annealing study failed to produce similar results in $\mathrm{CeRhRuSi}_{2}$, it is currently not possible to rule out a tiny amount of bond length disorder such as is observed in the unannealed $\mathrm{UCu}_{4} \mathrm{Pd}$ system $\left(\sim 0.00044(4) \AA^{2}[22]\right)$.

On the other hand, there are interesting differences between the $\mathrm{CeRhRuSi}_{2}$ and $\mathrm{UCu}_{4} \mathrm{Pd}$ systems. As just noted, there is no evidence of any kind for bond length disorder in the CeRhRuSi ${ }_{2}$ system. In addition, the KLDM suggests that it would take far more lattice disorder to create NFL behavior. Therefore this system is apparently much closer to being well ordered from a KLDM point of view than $\mathrm{UCu}_{4} \mathrm{Pd}$. This conclusion is similar to that reached by MacLaughlin et al. [18], in which it was noted that the low-frequency weight in the spin fluctuation spectrum of $\mathrm{CeRhRuSi}_{2}$ was much lower than in $\mathrm{UCu}_{4} \mathrm{Pd}$, and, in fact, was much more similar to that measured in better ordered systems, such as $\mathrm{YbRh}_{2} \mathrm{Si}_{2}$ and $\mathrm{CeNi}_{2} \mathrm{Ge}_{2}$.

\section{Acknowledgements}

We wish to thank S. J. L. Billinge and X. Qiu for advice on the PDF data analysis. Work at Lawrence Berkeley National Laboratory was supported by the Office of Science (OS), U.S. Department of Energy (DOE) under Contract No. DE-AC03-76SF00098. Work at Argonne National Laboratory was supported by the DOE/OS, Office of Basic Energy Science (OBES), under Contract No. W-31-109-Eng-38. X-ray absorption data were collected at the Stanford Synchrotron Radiation
Laboratory, a national user facility operated by Stanford University on the behalf of DOE/OBES. $\mathrm{X}$-ray diffraction data were collected at the BESSRC-CAT of the Advanced Photon Source.

\section{References}

[1] G. R. Stewart, Rev. Mod. Phys. 73 (2001) 797.

[2] O. Trovarelli et al., Phys. Rev. Lett. 85 (2000) 626.

[3] L. Shlyk et al., J. Phys.: Condens. Matter 11 (1999) 3525.

[4] C. H. Booth et al., Phys. Rev. B 69 (2004) 104430.

[5] C. Petrovic et al., J. Phys.:Condens. Matter 13 (2001) L337.

[6] O. O. Bernal, D. E. MacLaughlin, H. G. Lukefahr, B. Andraka, Phys. Rev. Lett. 75 (1995) 2023.

[7] E. Miranda, V. Dobrosavljević, G. Kotliar, Phys. Rev. Lett. 78 (1997) 290.

[8] A. H. Castro Neto, G. Castilla, B. A. Jones, Phys. Rev. Lett. 81 (1998) 3531; A. H. Castro Neto, B. A. Jones, Phys. Rev. B 62 (2000) 14975; E. Miranda, and V. Dobrosavljević, Phys. Rev. Lett. 86 (2001) 264.

[9] V. T. Rajan, Phys. Rev. Lett. 51 (1983) 308.

[10] W. A. Harrison, Elementary Electronic Structure, World Scientific, London, 1999, p. 606.

[11] G. B. Beni, P. M. Platzman, Phys. Rev. B 14 (1976) 1514.

[12] G. G. Li, F. Bridges, C. H. Booth, Phys. Rev. B 52 (1995) 6332.

[13] P. Estrela et al., Phys. Rev. B 63 (2001) 212409.

[14] Th. Proffen, S. J. L. Billinge, J. Appl. Crystallogr. 32 (1999) 572.

[15] E. G. Moshopoulou et al., Appl. Phys. A 74 [Suppl.] (2002) S895.

[16] M. Daniel et al., submitted.

[17] T. Graf et al., Phys. Rev. Lett. 78 (1997) 3769.

[18] D. E. MacLaughlin et al., Physica B 326 (2003) 381.

[19] B. H. Grier, J. M. Lawrence, V. Murgai, R. D. Parks, Phys. Rev. B 29 (1984) 2664.

[20] C. H. Booth et al., Phys. Rev. Lett. 81 (1998) 3960.

[21] E. D. Bauer et al., Phys. Rev. B 65 (2001) 245114.

[22] C. H. Booth et al., Phys. Rev. B 65 (2002) 245114.

[23] A. Weber et al., Phys. Rev. B 63 (2001) 205116. 